\title{
Lateral Supraorbital Approach
}

\author{
Juha Hernesniemi, Hugo Andrade-Barazarte, \\ Rosalia Duarte, Joseph Serrone, and Ferzat Hijazy
}

\subsection{Introduction}

The lateral supraorbital (LSO) approach is the most common craniotomy used in Helsinki by Professor Juha Hernesniemi. He has used the LSO approach for more than 30 years and in more than 6000 surgeries. This approach is a simpler, faster, and less invasive modification of the classically established pterional approach described by Yasargil. It provides excellent exposure of anterior fossa lesions, suprasellar lesions, and most anterior circulation aneurysms.

Like the pterional craniotomy, the skin incision is just behind the hairline. However, the incision for this approach is kept short and does not reach the zygoma inferiorly. In the LSO approach, the skin-muscle flap is opened as a single-layer flap, sparing the meticulous and laborious subfascial dissection of the pterional approach that puts the frontotemporal branches of the facial nerve at risk of injury. The temporalis muscle is split only in its superior aspect, decreasing postoperative pain and masticatory dysfunction. Additionally, it

J. Hernesniemi $(\bowtie) \cdot H$. Andrade-Barazarte

R. Duarte · F. Hijazy

Department of Neurosurgery,

Helsinki University Central Hospital,

Helsinki, Finland

e-mail: juha.hernesniemi@hus.fi

J. Serrone

Department of Neurosurgery, Virginia Mason

Medical Center, Seattle, WA, USA provides better cosmetic results due to less dissection of the temporalis muscle, which preserves its neurovascular supply.

\subsection{Steps of the Approach}

\subsubsection{Positioning}

The patient is positioned supine with the head and shoulders elevated above the cardiac level (Fig. 4.1) to reduce the bleeding by facilitating cerebral venous drainage. This in turn creates a "slack brain." The head is fixed with three or four pins to the head frame (Mayfield or Sugita) and is rotated $15-30^{\circ}$ toward the opposite side, flexed slightly laterally (Fig. 4.2), and either flexed or extended according to the location of the lesion to be approached. If the head is rotated too much toward the opposite side, the temporal lobe obstructs access into the Sylvian fissure. The degree of head flexion and extension depends on the craniocaudal distance between the pathology and the base of the anterior cranial fossa. Higher lesions require more extension of the head, whereas lesions located nearer to the skull base require slight flexion of the head. We use the Sugita's head frame because it provides a strong retraction force by the spring hooks and also allows the surgeon to rotate the head during the surgery. When this feature is not possible, the operating table can be rotated according to the surgeon needs. 


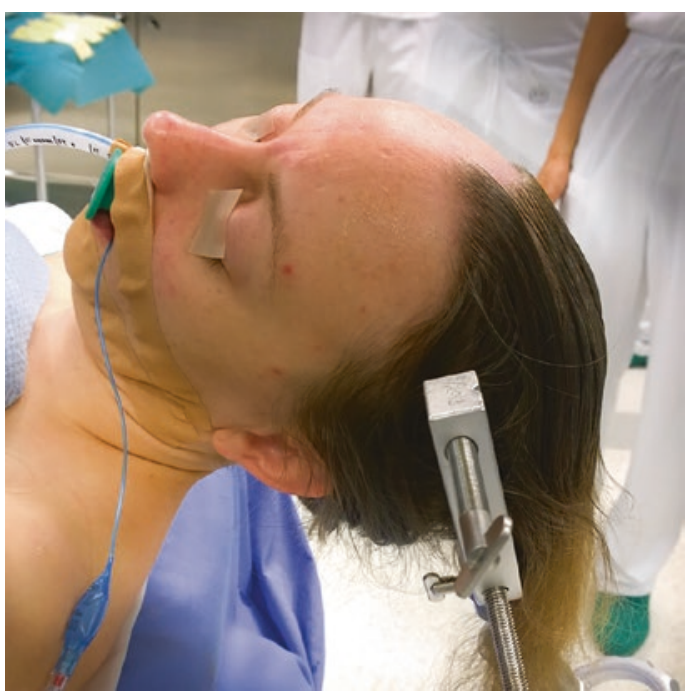

Fig. 4.1 Lateral view. The patient is positioned supine with the head and shoulders elevated above the cardiac level to reduce the bleeding

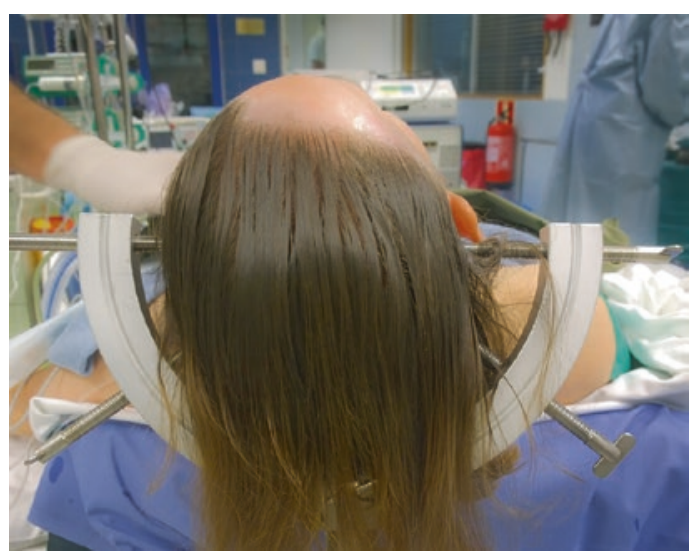

Fig. 4.2 The head is fixed with three or four pins to the Sugita's head frame and is rotated $15-30^{\circ}$ toward the opposite side and flexed slightly laterally

\subsubsection{Incision}

After minimal shaving, an oblique frontotemporal skin incision is made behind the hairline. The incision starts 3 centimeters $(\mathrm{cm})$ above the root of the zygoma and extends $2 \mathrm{~cm}$ medial to the

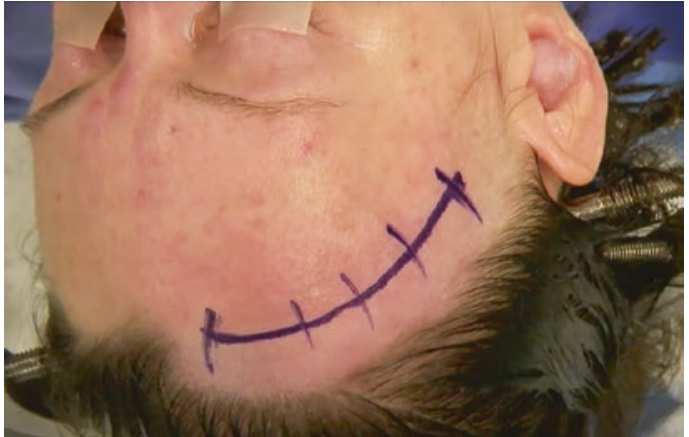

Fig. 4.3 Superior view. Oblique frontotemporal skin incision, behind the hairline



Fig. 4.4 The skin-galea-muscle flap elevated as a single layer and retracted anteriorly with spring hooks

ipsilateral midpupillary line (Fig. 4.3). In balding patients, the medial part of the incision can be placed in a skin wrinkle to provide better cosmetic results. The skin-galea-muscle flap is elevated in a single layer and retracted anteriorly with spring hooks until exposure of the superior orbital rim and frontal root of the zygoma. Raney clips are placed on the posterior margin of the skin incision for hemostasis (Fig. 4.4). The temporalis muscle is split only in its most superior and anterior part (only $3 \mathrm{~cm}$ of temporalis muscle is detached), and a spring hook is used to retract the muscle toward the zygomatic arc (Fig. 4.5). 


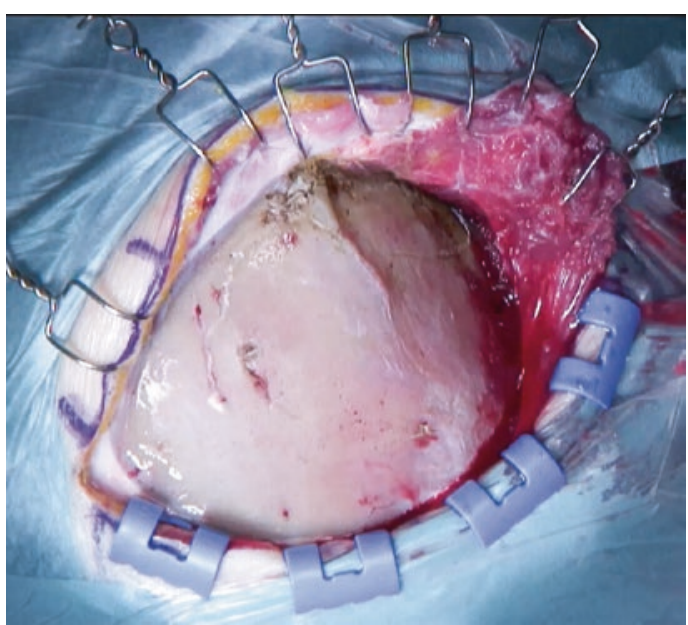

Fig. 4.5 Exposure of the superior orbital rim and frontal root of the zygoma. The temporalis muscle is detached only in its superior portion

\subsubsection{Craniotomy}

A single burr hole is placed under the most posteriorly exposed insertion line of the temporalis muscle. If necessary, another burr hole may be placed over the pterion, depending on the size of the flap, thickness of the bone, and adherence of the dura to the inner skull surface. A curved and stout dissector is used to detach the dura from the bone. Next, a side-cutting craniotome with a footplate is used to obtain an approximately $4 \mathrm{~cm} \times 4 \mathrm{~cm}$ bone flap with the Sylvian fissure located at the inferior limit of the craniotomy. The first cut starts from the burr hole and curves superiorly and then anteriorly toward the zygomatic process of the frontal bone. The second cut again starts in the burr hole and proceeds inferiorly toward the temporal bone, leaving the sphenoid ridge between these two cuts. The side-cutting craniotome without footplate is used to drill small holes around the craniotomy edges for future placement of dural tack-up sutures (Fig. 4.6). The thick frontal bone and lateral sphenoid ridge is thinned using the side-cutting craniotome. The bone flap is elevated posteriorly and cracked along the drilled line over the frontal and sphenoid bones.

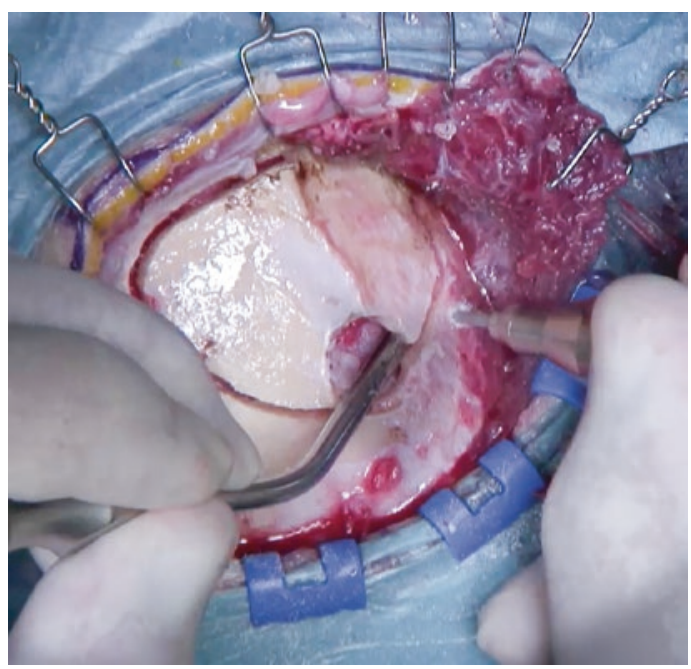

Fig. 4.6 A single burr hole is placed under the superior temporal line, and two cuts are made with the craniotome until obtaining a bone flap of $4 \times 4 \mathrm{~cm}$ approximately

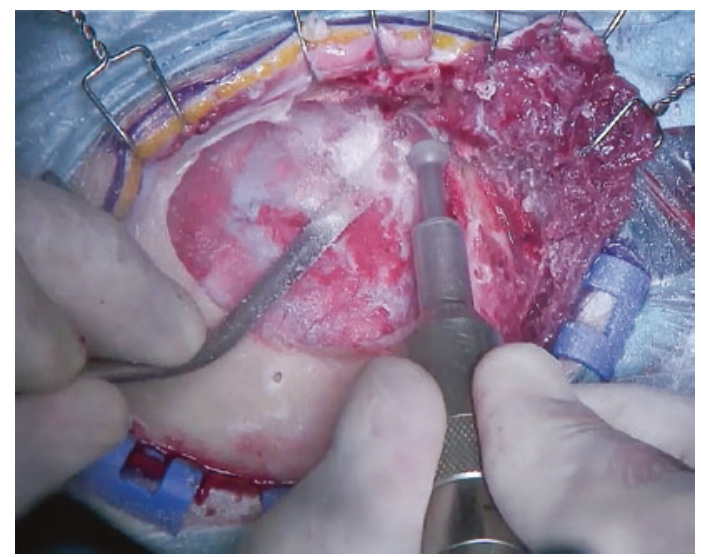

Fig. 4.7 Hot drilling technique of the sphenoid ridge and lateral and superior orbital wall

After the removal of the bone flap, the lateral sphenoid ridge is drilled off using first a high-speed drill with a rounded bit, starting at the lateral wall of the orbit toward the lesser sphenoid wing. Further drilling is continued with diamond-tipped drill bit without irrigation to smooth the surface and stop oozing of blood from the bone (Fig. 4.7). This socalled hot drilling technique (diamond-tipped drill 


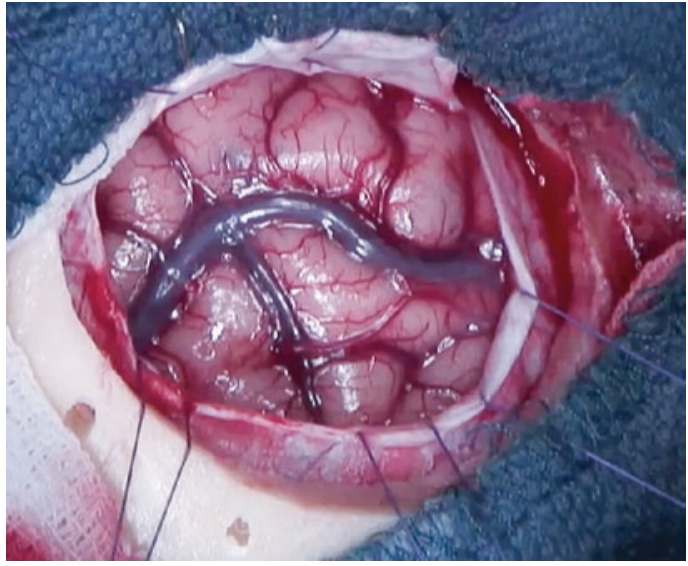

Fig. 4.8 The dura is opened in a curvilinear fashion and reflected anterolaterally. Multiple tack-up sutures are placed to retract the dural edges toward the craniotomy margins. White arrow and line demonstrated the place of the Sylvian Fissure

without saline irrigation heating the bone and sealing the bleeding) finishes the bone removal and offers excellent hemostasis. Before opening the dura, the area surrounding the craniotomy is covered with three hydrogen peroxide-soaked towels for antimicrobial effect. A green cloth is attached with staples at the anterior and inferior limit of the craniotomy to decrease the color balance in the operative field and improve visualization.

The dura is opened in a curvilinear fashion and reflected anterolaterally. Multiple tack-up sutures (Fig. 4.8) are placed to retract the dural edges toward the craniotomy margins preventing oozing from epidural space. At this point, the operating microscope is brought in place.

Before beginning the intracranial dissection, fibrin glue and small pieces of Surgicel ${ }^{\circledR}$ are used to achieve hemostasis and obtain a clear surgical field. Usually, the main goal of the initial subarachnoid dissection is to reach the basal cisterns to release cerebrospinal fluid (CSF) for brain relaxation. The dissection proceeds along the frontobasal surface of the frontal lobe slightly medial to the proximal Sylvian fissure until the entrance of the ipsilateral optic nerve into the optic canal. The arachnoid membranes limiting the optic cistern are opened and CSF is released. Furthermore, CSF can be released through the carotid cistern lateral to the ipsilateral optic nerve. Once a slack brain is achieved, the dissection pro- ceeds according to the pathology being treated. In cases of a very tight brain, CSF can also be released from the ventricles by opening of the lamina terminalis. To reach the lamina terminalis, the dissection continues from the ipsilateral optic nerve to the optic chiasm. The frontal lobe is retracted gently using tandem work of the suction and bipolar until the lamina terminalis is visualized, just posterior to the optic chiasm. The lamina terminalis is punctured with a sharp bipolar forceps or with microscissors allowing further drainage of CSF directly from the third ventricle.

In the LSO approach, the Sylvian fissure is located on the temporal edge of the craniotomy, and all the work to open the fissure is performed from the frontal side. An 18-guage sharp needle is used to open a small gap into the arachnoid membrane of the fissure. This small opening is filled with water (water dissection technique by Toth) to expand the subarachnoid space and facilitate a bloodless subarachnoid dissection. Then the dissection continues according to the pathology to be treated.

\subsubsection{Closure}

The complete closure is performed under high magnification of the operative microscope. The dura is closed tightly as much as possible, in a continuous fashion with a resorbable filament (Safil 4-0). Dural defects are sealed using hemostatic agents like $\mathrm{TachoSil}^{\circledR}$, fibrin glue, and Surgicel $^{\circledR}$. Careful and meticulous hemostasis is performed with bipolar coagulation. Additional tack-up sutures can be placed between the dura and the previously drilled holes around the craniotomy edges to elevate the dura and decrease the risk of postoperative epidural hematoma. At the final stage of the dural suturing, a blunt needle is inserted through a small gap between the dural margins, to fill the subdural space with saline and remove the air. After this, the gap is covered with TachoSil $^{\circledR}$, fibrin glue, and/or Surgicel ${ }^{\circledR}$.

The bone flap is replaced and fixed using two CranioFix $^{\circledR}$ clamps. The temporalis muscle is sutured using interrupted resorbable take-off filaments (Vicryl 2-0 or 1-0). The galea and subcutaneous layer are sutured simultaneously using a 
running suture with resorbable filament (Vicryl 3-0) without placing any drain. The skin is closed using surgical staples. However, in small children or young patients, the skin is closed with running resorbable intracutaneous suture (Monocryl 4-0). In some reoperations, the distance between the staples is less than $4-5 \mathrm{~mm}$ to ensure an uneventful wound healing.

\subsection{Indications of the Approach}

The LSO approach provides access to all anterior circulation aneurysms, except those located at the distal anterior cerebral artery. In addition, this approach can be used for high-positioned basilar bifurcation and basilar-superior cerebellar artery aneurysms. The trajectory of the approach provides an excellent view of the anterior fossa, the sellar and suprasellar regions, and the anterior part of the Sylvian fissure. Moreover, the LSO approach can be tailored more frontally or temporally depending of the location of a specific lesion.

\subsection{Limitation of the Approach}

This approach has certain limitations. It is not feasible for lesions requiring significant lateral (temporal) exposure. Examples of these cases include large posterior communicating artery aneurysms projecting posteriorly, large or giant middle cerebral artery aneurysms projecting laterally against the sphenoid wing, middle cerebral artery aneurysms associated with large temporoparietal intracerebral hematomas, or basilar tip aneurysms lying below the posterior clinoid process. In those cases, different approaches such as pterional, subtemporal, or a combination of both is necessary to treat the pathology.

\subsection{Complication and How to Avoid}

During the drilling of the sphenoid ridge, it is common to open the lateral and superior orbital wall causing extrusion of the periorbital fat into the surgical field. To solve this problem, we place a piece of $\mathrm{TachoSil}^{\circledR}$ over the defect. This keeps the periorbital fat contained while the bony work is completed. During the dural opening, we place two stiches at the inferior and anterior limit of the dural flap and retract this segment anteriorly using high tension. This maneuver keeps the periorbital fat away from the surgical field and provides $2-3 \mathrm{~mm}$ of extra space.

Since the LSO approach is a simple and less invasive approach, the rate of complications is less. CSF leakage represents a minor complication related to the approach. To decrease the risk of postoperative CSF leakage, we take special care during the dural closure placing small pieces of TachoSil ${ }^{\circledR}$, Surgicel $^{\circledR}$, and fibrin glue in the dural defect to obtain a complete closed surface. Due to the small size of the bone flap, the risk of an epidural hematoma is low. To further decrease this risk, we place multiple tack-up sutures between the dura and the small holes drilled around the craniotomy edges.

The LSO approach represents a fast and simple approach to treat a multitude of vascular lesions and tumors. This approach follows the basic principles described by the senior author (J.H): "Simple, clean, while preserving the normal anatomy."

\section{Bibliography}

1. Hernesniemi J, Ishii K, Karatas A, et al. Surgical technique to retract the tentorial edge during subtemporal approach: technical note. Neurosurgery. 2005;57(4 Suppl):E408; discussion E408.

2. Kivelev J, Hernesniemi J. Four-fold benefit of wound closure under high magnification. Surg Neurol Int. 2013;4:115.

3. Lehecka M, Laakso A, Hernesniemi J. Helsinki microneurosurgery basics and tricks. Germany: Aesculap AG; 2011.

4. Romani R, Elsharkawy A, Laakso A, Kangasniemi M, Hernesniemi J. Complications of anterior clinoidectomy through lateral supraorbital approach. World Neurosurg. 2012;77(5-6):698-703.

5. Romani R, Laakso A, Kangasniemi M, Niemela M, Hernesniemi J. Lateral supraorbital approach applied to tuberculum sellae meningiomas: experience with 52 consecutive patients. Neurosurgery. 2012;70(6):1504-18; discussion 1518-1509. 
Open Access This chapter is licensed under the terms of the Creative Commons Attribution 4.0 International License (http://creativecommons.org/licenses/by/4.0/), which permits use, sharing, adaptation, distribution and reproduction in any medium or format, as long as you give appropriate credit to the original author(s) and the source, provide a link to the Creative Commons license and indicate if changes were made.

The images or other third party material in this chapter are included in the chapter's Creative Commons license, unless indicated otherwise in a credit line to the material. If material is not included in the chapter's Creative Commons license and your intended use is not permitted by statutory regulation or exceeds the permitted use, you will need to obtain permission directly from the copyright holder.

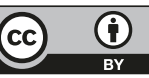

